Check for updates

Cite this: RSC Adv., 2019, 9, 30932

\title{
Impact of metallic trace elements on relaxivities of iron-oxide contrast agents
}

\author{
Ji Ma and Kezheng Chen (D) *
}

In this work, well-defined $3 \mathrm{~nm}$-sized $\mathrm{Ca}^{2+}, \mathrm{Fe}^{3+}, \mathrm{Na}^{+}, \mathrm{Mg}^{2+}, \mathrm{Zn}^{2+}, \mathrm{Ni}^{2+}, \mathrm{Co}^{2+}$, and $\mathrm{Cd}^{2+}$ cation-adsorbed $\mathrm{Fe}_{3} \mathrm{O}_{4} / \gamma-\mathrm{Fe}_{2} \mathrm{O}_{3}$ nanoparticles were used as prototype systems to investigate the influence of metallic trace elements in body fluids on the relaxivities of iron-oxide contrast agents. It was found that surfaceadsorbed cations formed a deterioration layer to induce pronounced relaxivity loss. Theoretical study showed that such relaxivity loss can be well described by a modified GCAS function, taking into account the harmonic cation oscillations around $\mathrm{Fe}_{3} \mathrm{O}_{4} / \gamma-\mathrm{Fe}_{2} \mathrm{O}_{3}$ nanoparticles. Quantum mechanics analyses revealed that even-parity and odd-parity states of harmonic oscillations are dominant in $r_{1}$ and $r_{2}$ relaxivities, respectively. Moreover, the harmonic oscillations of $\mathrm{Na}^{+}$and $\mathrm{Mg}^{2+}$ cations around $\mathrm{Fe}_{3} \mathrm{O}_{4} / \gamma^{-}$ $\mathrm{Fe}_{2} \mathrm{O}_{3}$ nanoparticles are found to be classical forbidden, which are quite different from their counterparts located in the classical permissive area.

Received 9th September 2019 Accepted 23rd September 2019

DOI: 10.1039/c9ra07227f

rsc.li/rsc-advances elements in complex fluid environments. This is an important but poorly understood matter open to discussion.

Given that these two tough issues are mainly related to the complicated in vivo environments, therefore, in this work, we study the influence of metallic trace elements in fluids on relaxivities of iron-oxide contrast agents. Basically, trace elements in human body can be divided into three types: (i) essential trace elements, including iodine, zinc, selenium, copper, molybdenum, chromium, cobalt, and iron elements; (ii) possibly essential trace elements, including manganese, silicon, boron, vanadium, and nickel elements; (iii) potentially toxic elements but essential in their low dose, including fluorine, lead, cadmium, mercury, arsenic, aluminium, and tin elements. In this study, $\mathrm{Fe}^{3+}, \mathrm{Zn}^{2+}, \mathrm{Co}^{2+}$ (type i), $\mathrm{Mg}^{2+}, \mathrm{Ni}^{2+}$ (type ii), $\mathrm{Cd}^{2+}$ (type iii) cations together with two macroelements $\left(\mathrm{Ca}^{2+}\right.$ and $\mathrm{Na}^{+}$cations) were chosen as typical elements to separately adsorb onto negatively charged $3 \mathrm{~nm}$-sized $\mathrm{Fe}_{3} \mathrm{O}_{4} / \gamma-\mathrm{Fe}_{2} \mathrm{O}_{3}$ contrast agents. Relaxivity measurements were then performed to demonstrate relaxivity loss and its variation rule after adsorption of various metallic cations.

\section{Results and discussion}

The XRD pattern of the as-synthesized pristine product in Fig. 1(a) shows the presence of $\mathrm{Fe}_{3} \mathrm{O}_{4}$ (JCPDS no. 85-1436) phase or $\gamma-\mathrm{Fe}_{2} \mathrm{O}_{3}$ (JCPDS no. 39-1346) phase, or both. Further, XPS spectrum in Fig. 1(b) gives two distinct peaks at $\sim 711$ and $\sim 724 \mathrm{eV}$ corresponding to $\mathrm{Fe} 2 \mathrm{p}_{3 / 2}$ and $\mathrm{Fe} 2 \mathrm{p}_{1 / 2}$ levels, respectively. ${ }^{17}$ Two satellite peaks around 718 and $732 \mathrm{eV}$ demonstrate the existence of $\gamma-\mathrm{Fe}_{2} \mathrm{O}_{3}$ phase, ${ }^{18}$ indicating that the pristine product is a hybrid of both $\mathrm{Fe}_{3} \mathrm{O}_{4}$ phase and $\gamma-\mathrm{Fe}_{2} \mathrm{O}_{3}$ phase. The

TEM study shows that pristine $\mathrm{Fe}_{3} \mathrm{O}_{4} @ \gamma-\mathrm{Fe}_{2} \mathrm{O}_{3}$ particles are
Lab of Functional and Biomedical Nanomaterials, College of Materials Science and Engineering, Qingdao University of Science and Technology, Qingdao 266042, China. E-mail: kchen@qust.edu.cn; Fax: +86-532-84022509; Tel: +86-532-84022509 
near spherical [inset of Fig. 1(a)], with an average diameter around $3 \mathrm{~nm}$. These $3 \mathrm{~nm}$-sized $\mathrm{Fe}_{3} \mathrm{O}_{4} @ \gamma-\mathrm{Fe}_{2} \mathrm{O}_{3}$ particles possess negatively charged surfaces with a zeta potential value of $-27.6 \mathrm{mV}$, which is of benefit to cation adsorption in aqueous solutions. Prior to the impregnation of these bare nanoparticles into various cation stock solutions, control experiments on relaxivities were carried out for these pristine $\mathrm{Fe}_{3} \mathrm{O}_{4} / \gamma-\mathrm{Fe}_{2} \mathrm{O}_{3}$ nanoparticles. Fig. 1(c) shows $r_{1}$ and $r_{2}$ relaxivities are determined (from slopes) to be 3.86 and $17.41 \mathrm{~s}^{-1} \mathrm{mmol}^{-1} \mathrm{~L}$, respectively, with an $r_{2} / r_{1}$ ratio of 4.51 . Although these values are barely comparable to Magnevist (a commercial gadolinium complex, $r_{1}=4.6 \mathrm{~s}^{-1} \mathrm{mmol}^{-1} \mathrm{~L}, r_{2}=4.5 \mathrm{~s}^{-1} \mathrm{mmol}^{-1} \mathrm{~L}, r_{2} / r_{1} \sim$ $1)^{\mathbf{1 9 , 2 0}}$ and much inferior to commercial Feridex $\left(r_{2}=109.4 \mathrm{~s}^{-1}\right.$ $\left.\mathrm{mmol}^{-1} \mathrm{~L}\right),{ }^{1}$ the optimization of relaxivities and development of new contrast agents are outside the scope of this work.
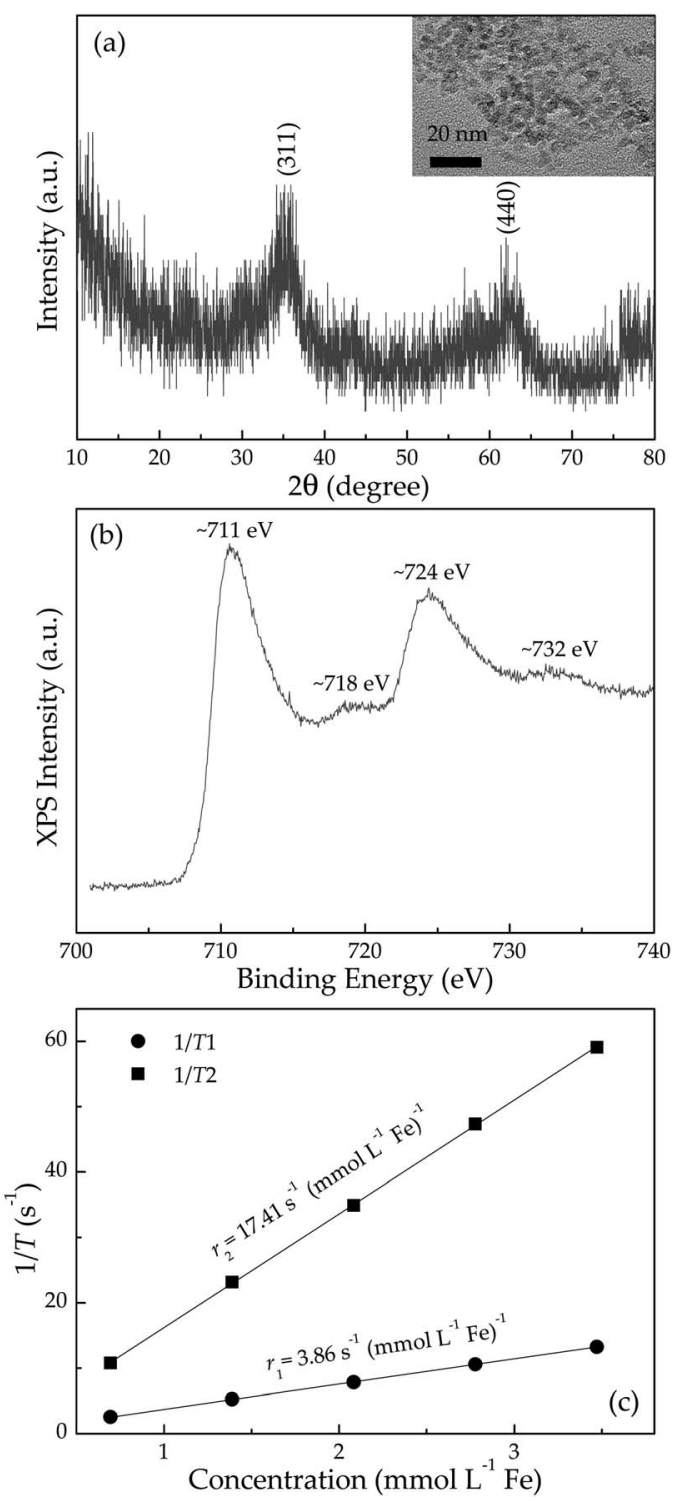

Fig. 1 (a) XRD pattern, TEM image (inset), (b) XPS spectrum and (c) proton longitudinal $r_{1}$ and transverse $r_{2}$ relaxivities (measured at $1.41 \mathrm{~T}$ and $37{ }^{\circ} \mathrm{C}$ ) of pristine $\mathrm{Fe}_{3} \mathrm{O}_{4} / \gamma-\mathrm{Fe}_{2} \mathrm{O}_{3}$ nanoparticles.
After various cation adsorptions, all $r_{1}$ relaxivities and most $r_{2}$ relaxivities drastically fall into decline (Fig. 2), indicating the degradation of MRI performance of $\mathrm{Fe}_{3} \mathrm{O}_{4} / \gamma-\mathrm{Fe}_{2} \mathrm{O}_{3}$ nanoparticles. That is to say, the inevitable adsorption of ions in body fluids in clinical application may well deteriorate the asdesigned MRI properties from in vitro tests. In this scenario, the quantitative research on the variation of $r_{1}$ and $r_{2}$ relaxivities after adsorption of various metallic cations is of fundamental importance to predicting MRI performance for in vivo applications.

Based on observations in Fig. 2, the surface-adsorbed metallic cations form a deterioration layer on relaxivities of $\mathrm{Fe}_{3} \mathrm{O}_{4} / \gamma-\mathrm{Fe}_{2} \mathrm{O}_{3}$ nanoparticles. Therefore, simplifications like independency between adsorbed cationic shell and interior iron-oxide core will not yield the accurate results and are full of fallacies. Along this line, it is essential to consider synergetic interactions between adsorbed cations and particle surfaces from a microscopic standpoint, and even to consider stochastic dependence between electrons and ions at a more microcosmic level. We can describe this idea more precisely in the following way: consider an electrostatically adsorbed cation vibrating at an iron-oxide particle
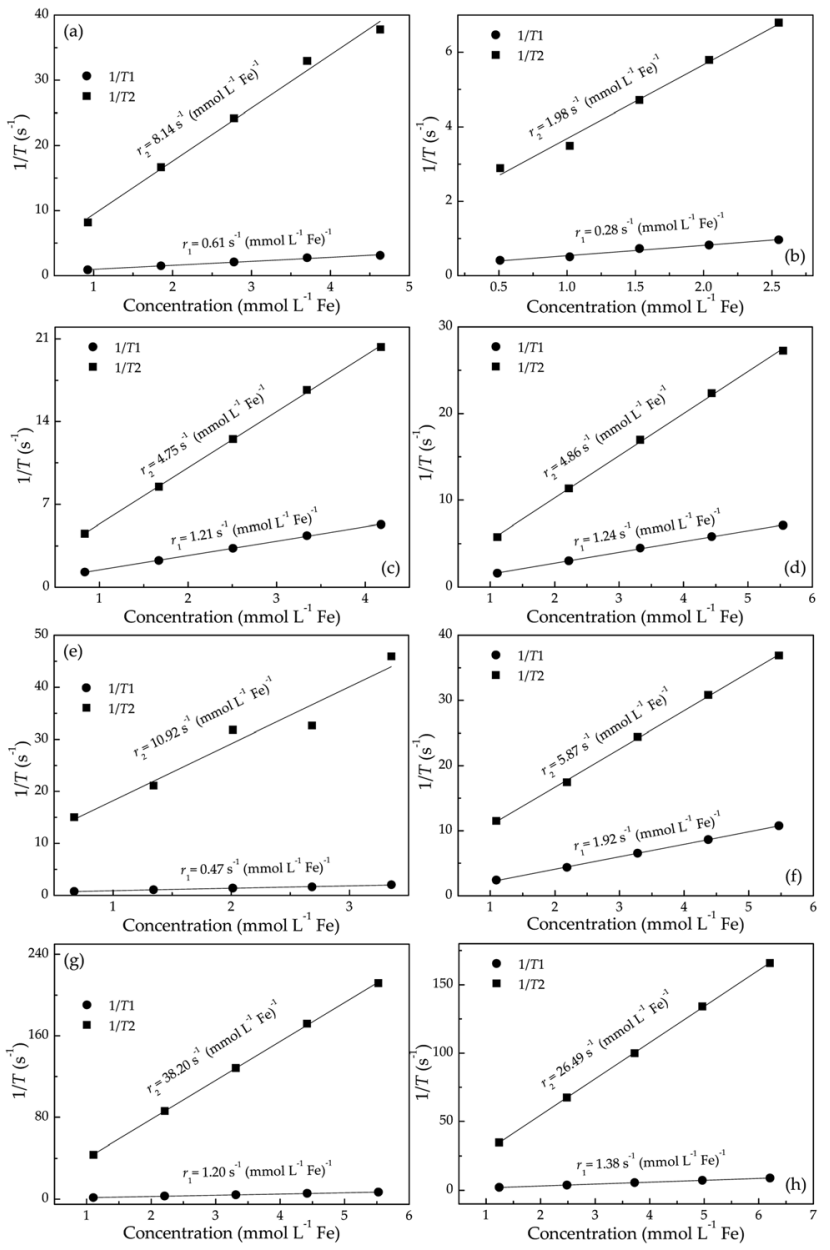

Fig. 2 Proton longitudinal $r_{1}$ and transverse $r_{2}$ relaxivities (measured at $1.41 \mathrm{~T}$ and $37^{\circ} \mathrm{C}$ ) of (a) $\mathrm{Ca}^{2+}$, (b) $\mathrm{Fe}^{3+}$, (c) $\mathrm{Na}^{+}$, (d) $\mathrm{Mg}^{2+}$, (e) $\mathrm{Zn}^{2+}$, (f) $\mathrm{Ni}^{2+}$, (g) $\mathrm{Co}^{2+}$, and (h) $\mathrm{Cd}^{2+}$ adsorbed $\mathrm{Fe}_{3} \mathrm{O}_{4} / \gamma-\mathrm{Fe}_{2} \mathrm{O}_{3}$ nanoparticles. 
surface. It becomes common sense that any three-dimensional vibration can always be decomposed into numerous onedimensional harmonic vibrations. In this sense, we draw lessons from conclusions in quantum mechanics and use Hermite polynomials, which belong to a classical orthogonal polynomial sequence, to make the association between final relaxivities and a defined parameter $X r / Z$ of adsorbed cations. Here, $X, r$ and $Z$ denote electronegativity, radius and atomic number of the cation under discussed (Table 1), respectively. The constructed function of $\mathrm{Xr} / \mathrm{Z}$-dependent relaxivity distribution is based on the probability density function utilized in GramCharlier A series (GCAS) with some modifications. As known, GCAS function can also be expressed by using Hermite polynomials but it is not guaranteed to be positive, and is therefore not a valid probability distribution. It does not converge in many cases of interest, making it not a true asymptotic expansion, because it is not possible to estimate the error of the expansion. Based on the preceding analyses, the relaxivities $r_{k}(k=1$ or 2$)$ can be given by

$$
r_{k}=r_{k 0}+\frac{A}{w \sqrt{2 \pi}} \mathrm{e}^{-z^{2} / 2}\left(1+\left|\sum_{i=0}^{4} \frac{a_{i}}{i !} H_{i}(z)\right|\right)
$$

wherein $z$ has an expression of $\left(x-x_{c}\right) / w(x=X r / Z), a_{i}$ are cumulants and $A, w$ are constants. The low-order Hermite polynomials involved in eqn (1) are $H_{0}=1, H_{1}=2 z, H_{2}=4 z^{2}-$ 2, $H_{3}=z^{3}-3 z$, and $H_{4}=z^{4}-6 z^{3}+3$.

By applying eqn (1), the experimental data of both $r_{1}$ and $r_{2}$ relaxivities for various adsorbed cations can be separately connected with smooth curves, as shown in Fig. 3(a) and (b), respectively. The correlation coefficient $R^{2}$ values of the fitting plots are beyond 0.95 , indicating the applicability of eqn (1) to make an association between relaxivities and the characteristic parameter $X r / Z$ of adsorbed cations. Most strikingly, the cumulants $a_{i}$ are close to zero at $i=1,3$ in longitudinal case and at $i=0,2,4$ in transverse case (Table 2). This intriguing finding reminds us of the parity of Hermite polynomials, that is, $H_{i}(-z)$ $=(-1)^{i} H_{i}(z)$. Along this line, we boldly propose a general formula of cumulants $a_{i}$ according to the harmonic oscillator theory in quantum mechanics:

$$
a_{i}=\sqrt{\frac{\alpha i !}{\sqrt{\pi} 2^{i}}}
$$

Here, $\alpha$ denotes the coefficient of $x$ in the expression of $z$. Based on eqn (2), the values of $a_{0}, a_{2}$, and $a_{4}$ are calculated to be about $0.75,0.53$ and 0.92 , respectively, and the values of $a_{1}$ and $a_{3}$ are calculated to be 0.53 and 0.65 , respectively. Obviously, these

Table 1 Electronegativity, radius and atomic number of the adsorbed

\begin{tabular}{|c|c|c|c|c|c|c|c|c|}
\hline Parameters & $\mathrm{Ca}^{2+}$ & $\mathrm{Fe}^{3+}$ & $\mathrm{Na}^{+}$ & $\mathrm{Mg}^{2+}$ & $\mathrm{Zn}^{2+}$ & $\mathrm{Ni}^{2+}$ & $\mathrm{Co}^{2+}$ & $\mathrm{Cd}^{2+}$ \\
\hline Atomic number & 20 & 26 & 11 & 12 & 30 & 28 & 27 & 48 \\
\hline Electronegativity & 11.30 & 15.38 & 9.44 & 17.13 & 10.38 & 9.60 & 9.10 & 9.79 \\
\hline Radius $(\AA)$ & 0.99 & 0.64 & 0.95 & 0.65 & 0.74 & 0.72 & 0.74 & 0.97 \\
\hline
\end{tabular}
cations
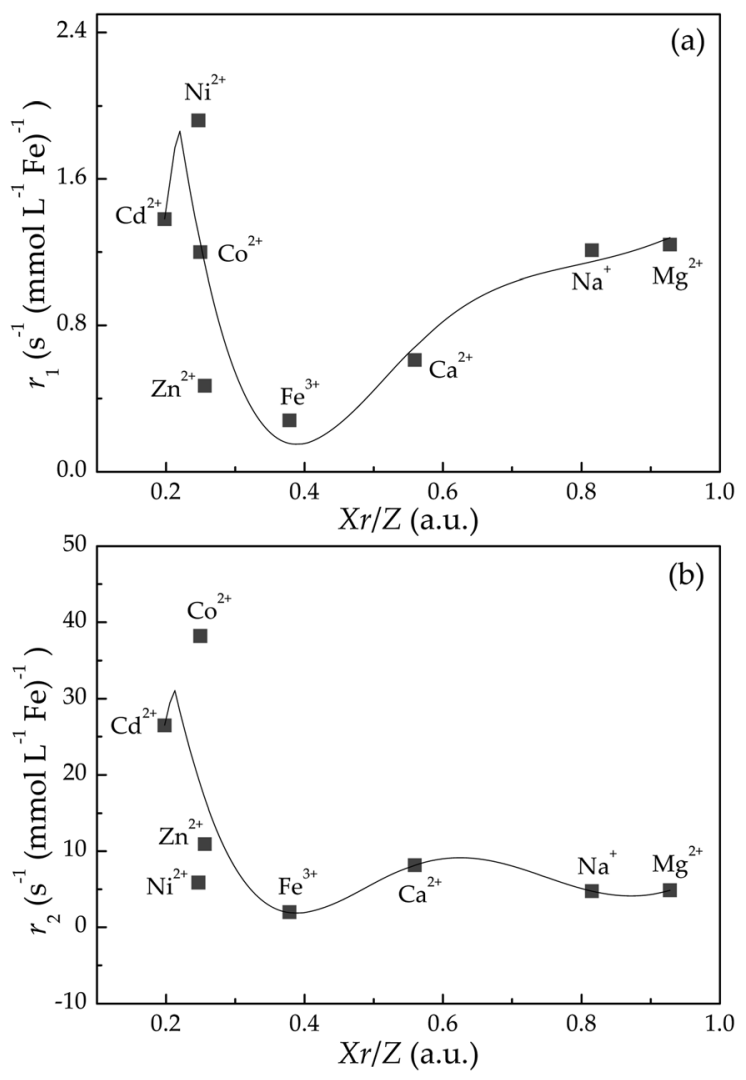

Fig. 3 Dependence of (a) $r_{1}$ and (b) $r_{2}$ values on $X r / Z$ with regard to various cation-adsorbed $\mathrm{Fe}_{3} \mathrm{O}_{4} / \gamma-\mathrm{Fe}_{2} \mathrm{O}_{3}$ nanoparticles. The experimental data are plotted as solid squares, and the solid lines are fitting results according to eqn (1).

calculated values are in excellent consistency with the corresponding fitting values in Table 2, and further validating the applicability of harmonic oscillator theory in our system. In this scenario, the nearly null value of $a_{1}$ and $a_{3}$ in longitudinal case reveals that the even-parity states of harmonic oscillators are dominant. Likewise, the odd-parity states of harmonic oscillators determines the transverse relaxivities due to $a_{0} \approx a_{2} \approx a_{4}$ $\approx 0$.

In addition, the quantum mechanics gives the characteristic length (i.e., $1 / \alpha)$ dividing classical forbidden area $(|z|>1 / \alpha)$ and classical permissive area $(|z| \leq 1 / \alpha)$ for harmonic oscillators. In this work, $z$ values can be calculated based on the fitting values of $x_{\mathrm{c}}$ and $w$ according to the equation of $z=\left(X r / Z-x_{\mathrm{c}}\right) / w$. Fig. 4 shows that $z$ values of $\mathrm{Na}^{+}$and $\mathrm{Mg}^{2+}$ cations are beyond $1 / \alpha=1$, indicating that the harmonic oscillations of $\mathrm{Na}^{+}$and $\mathrm{Mg}^{2+}$ cations around $\mathrm{Fe}_{3} \mathrm{O}_{4} / \gamma-\mathrm{Fe}_{2} \mathrm{O}_{3}$ nanoparticles are classical forbidden. By contrast, $\mathrm{Ca}^{2+}, \mathrm{Fe}^{3+}, \mathrm{Zn}^{2+}, \mathrm{Ni}^{2+}, \mathrm{Co}^{2+}$, and $\mathrm{Cd}^{2+}$ cations can oscillate around $\mathrm{Fe}_{3} \mathrm{O}_{4} / \gamma-\mathrm{Fe}_{2} \mathrm{O}_{3}$ nanoparticles within classical permissive area.

\section{Experimental}

\section{Synthesis and characterization}

The pristine $\mathrm{Fe}_{3} \mathrm{O}_{4} / \gamma-\mathrm{Fe}_{2} \mathrm{O}_{3}$ nanoparticles were synthesized according to the method reported elsewhere ${ }^{21,22}$ with little 
Table 2 Fitting parameters according to eqn (1)

\begin{tabular}{|c|c|c|c|c|c|c|c|c|c|}
\hline Relaxivity & $r_{k 0}$ & $A$ & $w$ & $x_{\mathrm{c}}$ & $a_{0}$ & $a_{1}$ & $a_{2}$ & $a_{3}$ & $a_{4}$ \\
\hline Longitudinal & 3.95 & $-1.6 \times 10^{-3}$ & 0.37 & 0.26 & 0.73 & $4.5 \times 10^{-5}$ & 0.53 & $2.5 \times 10^{-6}$ & 0.89 \\
\hline Transverse & 17.86 & -19.33 & 0.38 & 0.29 & $1.2 \times 10^{-3}$ & 0.58 & $1.7 \times 10^{-6}$ & 0.66 & $8.9 \times 10^{-8}$ \\
\hline
\end{tabular}

modification. Typically, $10 \mathrm{~mL}$ of $\mathrm{Na}_{2} \mathrm{CO}_{3}$ aqueous solution $\left(0.6 \mathrm{~mol} \mathrm{~L}^{-1}\right)$ was added into $50 \mathrm{~mL}$ of $\mathrm{FeCl}_{3} \cdot 6 \mathrm{H}_{2} \mathrm{O}$ aqueous solution $\left(0.04 \mathrm{~mol} \mathrm{~L}^{-1}\right)$ and vigorously stirred for five minutes. After that, ascorbic acid ( $0.5 \mathrm{~g}$ ) was added into the above solution and stirred for another ten minutes. Finally, the mixture was transferred into a Teflon-lined stainless-steel autoclave with a capacity of $100 \mathrm{~mL}$ for hydrothermal treatment at $160^{\circ} \mathrm{C}$ for $3 \mathrm{~h}$. After the autoclave had cooled down to room temperature naturally, the $\mathrm{Fe}_{3} \mathrm{O}_{4} / \gamma-\mathrm{Fe}_{2} \mathrm{O}_{3}$ precipitate was separated by centrifugation, washed successively with distilled water and absolute ethanol, and dried in the air at $60{ }^{\circ} \mathrm{C}$ for $4 \mathrm{~h}$.

To mimic the actual element concentration in human body, the concentration of $\mathrm{Ca}^{2+}, \mathrm{Fe}^{3+}, \mathrm{Na}^{+}, \mathrm{Mg}^{2+}, \mathrm{Zn}^{2+}, \mathrm{Ni}^{2+}, \mathrm{Co}^{2+}$, and $\mathrm{Cd}^{2+}$ cations was uniformly set to be $2 \mathrm{mmol} \mathrm{L}^{-1}$. In a typical adsorption experiment, eight sets of $50 \mathrm{mg}$ of the as-prepared $\mathrm{Fe}_{3} \mathrm{O}_{4} / \gamma-\mathrm{Fe}_{2} \mathrm{O}_{3}$ nanoparticles were separately soaked into $100 \mathrm{~mL}$ of $\mathrm{CaCl}_{2}, \mathrm{FeCl}_{3}, \mathrm{NaCl}, \mathrm{MgCl}_{2}, \mathrm{ZnCl}_{2}, \mathrm{NiCl}_{2}, \mathrm{CoCl}_{2}$, and $\mathrm{CdCl}_{2}$ aqueous solutions for $1 \mathrm{~h}$. After the above adsorption, all precipitates were separated by centrifugation, washed successively with distilled water and absolute ethanol, and dried in the air at $60{ }^{\circ} \mathrm{C}$ for $4 \mathrm{~h}$.

The X-ray diffraction (XRD) pattern of the sample was recorded with a Rigaku D/max- $\gamma \mathrm{B}$ diffractometer equipped with a rotating anode and a $\mathrm{Cu} \mathrm{K} \alpha$ source $(\lambda=0.154056 \mathrm{~nm})$ at $40 \mathrm{kV}$ and $150 \mathrm{~mA}$ between $10^{\circ}$ and $80^{\circ}(2 \theta)$ with a step size of $0.02^{\circ}$. The phase composition was further confirmed by X-ray photoelectron spectroscopy (XPS), which was performed on VG ESCALAB 220i-XL system. The morphology and structure of the sample was characterized by transmission-electron microscope (TEM) with an operating voltage of $200 \mathrm{kV}$ (JEM-2100). The zeta potential values of the samples were determined by dynamic

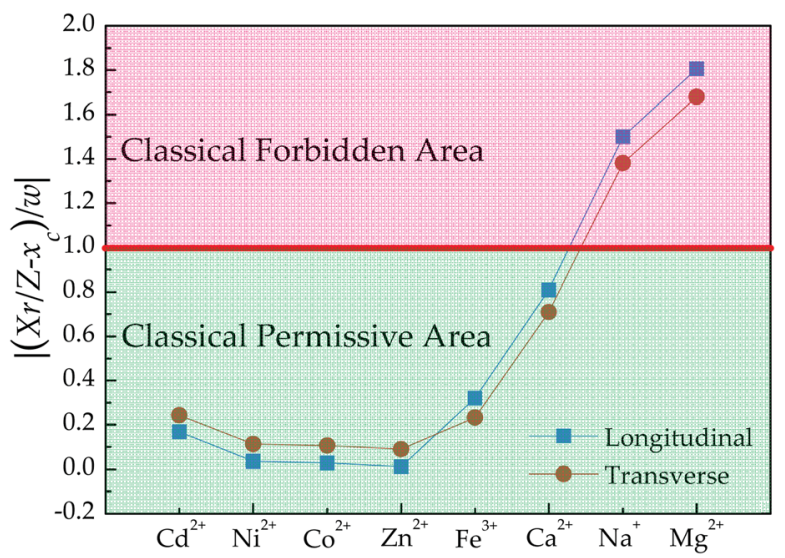

Fig. 4 Dependence of $\left(X r / Z-x_{c}\right) / w$ values for various cation-adsorbed $\mathrm{Fe}_{3} \mathrm{O}_{4} / \gamma-\mathrm{Fe}_{2} \mathrm{O}_{3}$ nanoparticles. light scattering (DLS, Zetasizer Nano, Malvern Instrument). Iron concentrations in relaxivity experiments were determined by inductively coupled plasma-optic emission spectrometry (ICPOES, PerkinElmer Optima 8000).

\section{Relaxivity measurement}

A mq-60 NMR analyzer (Bruker Minispec) was applied to measure proton transverse $\left(r_{2}\right)$ and longitudinal $\left(r_{1}\right)$ relaxivities of the samples. Specifically, a certain amount of sample was dissolved in deionized water at $37{ }^{\circ} \mathrm{C}$ and laid in a magnetic field of $1.41 \mathrm{~T}$ with a frequency of $60 \mathrm{MHz}$. Then a Carr-PurcellMeiboom-Gill spin-echo pulse sequence was adopted and the interecho time and repetition time were set to be $1 \mathrm{~ms}$ and $6 \mathrm{~s}$, respectively. The as-measured signal data was fitted by a monoexponential decay curve to yield the $T_{2}$ relaxation time. On the other hand, an inversion recovery (IR) pulse sequence was adopted by using 10 inversion times between 0.05 and $10 \mathrm{~s}$. Then a monoexponential recovery curve was utilized to fit the signal data to finally obtain $T_{1}$ relaxation time. All data were presented as arithmetic means of 3 replicates. After the measurements of $T_{1}$ and $T_{2}$, the relaxivities $r_{1}$ and $r_{2}$ could be obtained by fitting respective relaxation rate $\left(\mathrm{s}^{-1}\right)$ versus iron concentration $\left(\mathrm{mmol} \mathrm{L}^{-1} \mathrm{Fe}\right)$.

\section{Conclusions}

In summary, $\mathrm{Ca}^{2+}, \mathrm{Fe}^{3+}, \mathrm{Na}^{+}, \mathrm{Mg}^{2+}, \mathrm{Zn}^{2+}, \mathrm{Ni}^{2+}, \mathrm{Co}^{2+}$, and $\mathrm{Cd}^{2+}$ cation-adsorbed $\mathrm{Fe}_{3} \mathrm{O}_{4} / \gamma-\mathrm{Fe}_{2} \mathrm{O}_{3}$ nanoparticles are synthesized by a facile soaking method to mimic actual element adsorption in human body. The $r_{1}$ and $r_{2}$ relaxivities of bare $\mathrm{Fe}_{3} \mathrm{O}_{4} / \gamma-\mathrm{Fe}_{2} \mathrm{O}_{3}$ nanoparticles are 3.86 and $17.41 \mathrm{~s}^{-1} \mathrm{mmol}^{-1} \mathrm{~L}$, respectively. However, after cation adsorption, nearly all relaxivity values show a great decrease, manifesting surface-adsorbed cations greatly degenerate the MRI capability of iron-oxide contrast agents. Such relaxivity loss can be described by a modified GCAS function, taking into account of numerous harmonic oscillations of various cations around $\mathrm{Fe}_{3} \mathrm{O}_{4} / \gamma-\mathrm{Fe}_{2} \mathrm{O}_{3}$ nanoparticles. The quantum mechanics analyses show that even-parity and odd-parity states of harmonic oscillators are dominant in $r_{1}$ and $r_{2}$ relaxivities of cation-adsorbed $\mathrm{Fe}_{3} \mathrm{O}_{4} / \gamma-\mathrm{Fe}_{2} \mathrm{O}_{3}$ nanoparticles, respectively. Moreover, the harmonic oscillations of $\mathrm{Na}^{+}$and $\mathrm{Mg}^{2+}$ cations around $\mathrm{Fe}_{3} \mathrm{O}_{4} / \gamma-\mathrm{Fe}_{2} \mathrm{O}_{3}$ nanoparticles are found to be classical forbidden, which are quite different from their counterparts located in the classical permissive area.

\section{Conflicts of interest}

There are no conflicts to declare. 


\section{Acknowledgements}

The authors are grateful to the financial aid from the National Natural Science Foundation of China (NSFC No. 51472133).

\section{Notes and references}

1 T. Shin, J. Choi, S. Yun, I. Kim, H. Song, Y. Kim, K. I. Park and J. Cheon, ACS Nano, 2014, 8, 3393.

2 J. Y. Park, M. J. Baek, E. S. Choi, S. Woo, J. H. Kim, T. J. Kim, J. C. Jung, K. S. Chae, Y. Chang and G. H. Lee, ACS Nano, 2009, 3, 3663.

3 Q. Wang, A. Xiao, Y. Liu, Q. Zou, Q. Zhou, H. Wang, X. Yang, C. Zheng, Y. Yang and Y. Zhu, Nanomedicine, 2018, 14, 2551.

4 Y. Li, Y. Chang, R. Yuan and Y. Chai, ACS Appl. Mater. Interfaces, 2018, 10, 25213.

5 I. Monaco, P. Armanetti, E. Locatelli, A. Flori, M. Maturi, S. Del Turco, L. Menichetti and M. C. Franchini, J. Mater. Chem. B, 2018, 6, 2993.

6 Z. Zhao, C. Sun, J. Bao, L. Yang, R. Wei, J. Cheng, H. Lina and J. Gao, J. Mater. Chem. B, 2018, 6, 401.

7 H. B. Na, I. C. Song and T. Hyeon, Adv. Mater., 2009, 21, 2133. 8 Q. A. Pankhurst, J. Connolly, S. K. Jones and J. Dobson, J. Phys. D: Appl. Phys., 2003, 36, R167.

9 H. U. Ahmed, A. Kirkham, M. Arya, R. Illing, A. Freeman, C. Allen and M. Emberton, Nat. Rev. Clin. Oncol., 2009, 6, 197.

10 J. W. M. Bulte and D. L. Kraitchman, NMR Biomed., 2004, 17, 484.
11 W. Liu, H. Dahnke, E. K. Jordan, T. Schaeffter and J. A. Frank, NMR Biomed., 2008, 21, 242.

12 C. A. Helms, Am. J. Roentgenol., 1999, 173, 234.

13 W. S. Seo, J. H. Lee, X. Sun, Y. Suzuki, D. Mann, Z. Liu, M. Terashima, P. C. Yang, M. V. McConnell and D. G. Nishimura, Nat. Mater., 2006, 5, 971.

14 C. Richard, B. T. Doan, J. C. Beloeil, M. Bessodes, E. Tóth and D. Scherman, Nano Lett., 2008, 8, 232.

15 J. Shin, R. M. Anisur, M. K. Ko, G. H. Im, J. H. Lee and I. S. Lee, Angew. Chem., Int. Ed., 2009, 48, 321.

16 J. S. Choi, J. H. Lee, T. H. Shin, H. T. Song, E. Y. Kim and J. Cheon, J. Am. Chem. Soc., 2010, 132, 11015.

17 Z. Li, J. F. Godsell, J. P. O'Byrne, N. Petkov, M. A. Morris, S. Roy and J. D. Holmes, J. Am. Chem. Soc., 2010, 132, 12540. 18 T. Yamashita and P. Hayes, Appl. Surf. Sci., 2008, 254, 2441. 19 T. Kim, E. Momin, J. Choi, K. Yuan, H. Zaidi, J. Kim, M. Park, N. Lee, M. T. McMahon, A. Quinones-Hinojosa, J. W. M. Bulte, T. Hyeon and A. A. Gilad, J. Am. Chem. Soc., 2011, 133, 2955.

20 W. A. Seo, J. H. Lee, X. Sun, Y. Suzuki, D. Mann, Z. Liu, M. Terashima, P. C. Yang, M. V. McConnell, D. G. Nishimura and H. Dai, Nat. Mater., 2006, 5, 971.

21 J. Ma, L. Cheng and K. Chen, J. Appl. Phys., 2015, 117, 154701.

22 J. Ma and K. Chen, Sci. Rep., 2017, 7, 42312. 